\title{
Gestão estratégica de pessoas no teletrabalho durante a pandemia da COVID-19
}

\section{Strategic people management in teleworking during the COVID-19 pandemic}

Silvia Rocha de Souza ${ }^{1}$, Rita de Cassia de Marchi Barcelos Dalri², Marcia Eiko

Karino $^{3}$, Beatriz Maria dos Santos Santiago Ribeiro ${ }^{4}$

1. ORCID: https://orcid.org/0000-0003-1495-262X Enfermeira. Especialista em nefrologia, pós-graduanda em gestão de pessoas na Faculdade Nova Venda do Imigrante(FAVENI). E-mail: silviaeadrean@hotmail.com.

2. ORCID: https://orcid.org/0000-0002-6575-5426. Doutora. Docente do Curso de da Pós-Graduação Stricto Sensu Escola de Enfermagem de Ribeirão Preto (Universidade de São Paulo, Ribeirão Preto, São Paulo, Brasil). E-mail: ritacmbdalri@bol.com.br

3. ORCID: https://orcid.org/0000-0002-6582-2801. Doutora em Enfermagem. Docente do curso de graduação em enfermagem da Universidade Estadual de Londrina (UEL). Coordenadora da residência em urgência e Emergência da UEL, Londrina-ParanáBrasil. E-mail: marciak2503@hotmail.com.

4. ORCID: https://orcid.org/0000-0001-5211-5422. Mestre em Enfermagem. Doutoranda em Ciências da Saúde na Escola de Enfermagem (Universidade de São Paulo, Ribeirão Preto, São Paulo, Brasil). E-mail: beatrizsantiago1994@hotmail.com.

CONTATO: Nome do autor correspondente: Beatriz Maria dos Santos Santiago Ribeiro | Endereço: Sítio São Joaquim. Laranjal, Califórnia- PR. Telefone: (43) 9.91724895 - E-mail: beatrizsantiago1994@hotmail.com 
RESUMO A gestão estratégica de pessoas surgiu como um processo necessário para atender novas demandas. Objetivou-se neste estudo analisar as evidências científicas disponíveis na literatura nacional e internacional sobre a gestão de pessoas atuantes no teletrabalho desenvolvido diante das medidas preventivas impostas durante a pandemia da COVID-19. Estudo de exploração qualitativa de dados da literatura, desenvolvido entre os meses de abril e junho de 2020, sendo selecionadas as bases de dados: PUBMED, Literatura Latino-Americana e do Caribe em Ciências da Saúde, Physiotherapy Evidence Database e a Biblioteca Eletrônica Científica Online. Apenas dois estudos se relacionaram ao objetivo, ambos eram internacionais e evidenciaram a gestão estratégica de pessoas no teletrabalho de profissionais de saúde. A gestão foi implementada no teletrabalho adaptando-se ás ferramentas digitais e tecnológicas para a atividade laboral e atingindo resultados positivos. Essa gestão estratégica proporcionou o atendimento da demanda apresentada, preservando os cuidados de isolamento social preconizado.

DESCRITORES:Gestão de pessoas. Teletrabalho.COVID-19.

ABSTRACT Strategic people management emerged as a necessary process to meet new demands. The objective of this study was to analyze the scientific evidence available in the national and international literature on the people management working in telework developed in view of the preventive measures imposed during the pandemic of COVID-19. Qualitative exploration study of literature data, developed between April and June 2020, with the following databases being selected: PUBMED, Latin American and Caribbean Literature in Health Sciences, Physiotherapy Evidence Database and the Electronic Library Scientific Online. Only two studies were related to the objective, both were international and showed the strategic people management in teleworking by health professionals. Management was implemented in teleworking, adapting to digital and technological tools for work activity and achieving positive results. This strategic management made it possible to meet the demand presented, preserving the recommended social isolation care.

DESCRIPTORS: People management. Teleworking. COVID-19. 


\section{INTRODUÇÃO}

1 tualmente o mundo tem vivenciado a pandemia da COVID-19, doença causada pelo vírus SARS-COV-2 (vírus da síndrome respiratória aguda grave 2$)^{1}$. Os coronavírus fazem parte de uma grande família de vírus que causam doenças em pessoas;se originou em morcegos e foi transmitido aos seres humanos através de animais intermediários ainda em invetigação ${ }^{2-3}$. O primeiro caso da doença foi identificado na cidade de Wuhan, província de Hubei na China ${ }^{4-6}$.

A doença COVID-19 é transmitida por inalação ou contato com gotículas infectadas e o período de incubação varia de dois a 14 dias. Os sintomas mais comuns apresentados pelos indivíduos infectados são febre, tosse, dor de garganta, falta de ar, fadiga e malestar. A doença COVID-19 se manifesta de forma leve na maioria das pessoas; entretanto, em idosos e pessoas com comorbidades e outras doenças já instaladas pode evoluir para pneumonia, síndrome do desconforto respiratório agudo (SDRA) e disfunção de múltiplos órgãos ${ }^{7-8}$, levando a um estado de saúde grave que pode levar a morte ${ }^{9}$. Tem-se verificado também que muitas pessoas contaminadas podem apresentar-se assintomáticas.

A gestão estratégica de pessoas surgiu como um processo necessário para atender novas demandas consequente das transformações econômicas e culturais dos setores laborais públicos e privados. Sendo assim, a equipe gestora possui atribuições e competências para impulsionar os trabalhadores a trabalharem motivados, dispostos e valorizados; esse processo não é uma tarefa simples ${ }^{10}$. No cenário atual da pandemia da COVID-19 pode ser um desafio, já que a equipe de gestão de pessoas necessitou imediatamente de medidas emergenciais para prevenir as pessoas do contágio.

As medidas restritivas durante a pandemia da COVID-19, causaram grandes mudanças nos ambientes laborais. Assim, alguns profissionais deixaram seu ambiente laboral de costume, mas os que se enquadravam nos ditos trabalhadores essenciais se mantiveram trabalhando e outros ainda, aderiram ao teletrabalho, incluindo o setor de gestão de pessoas. No contexto de prevenção de propagação de doenças infecciosas, o teletrabalho passou a ser uma boa estratégia de conforto durante pandemias de gripe, quando as pessoas doentes passaram a trabalhalhar remotamente, assim evitaram a exposição de colegas de trabalho durante o período contagioso ${ }^{11}$.

Estudo realizado em 64 cidades chinesas com 369 indivíduos, avaliou a saúde e o bem-estar de adultos que viviam e trabalhavam após um mês de confinamento decorrente do surto da COVID-19. Os resultados encontrados mostraram que os participantes que trabalhavam no teletrabalho tinham melhor saúde mental do que aqueles que pararam de trabalhar. E em uma análise mais aprofundada das oito dimensões da escala avaliada, em comparação com as pessoas que retomaram o trabalho no escritório, aquelas que pararam totalmente de trabalhar demonstraram uma queda no estado geral de saúde, 
especialmente em relação a saúde mental e aumento das limitações, referentes a problemas físicos e emocionais ${ }^{12}$. Desse modo, o teletrabalho pode ser uma ferramenta que auxilia a saúde mental, devendo ser observado pela equipe de gestão de pessoas.

Evidências apoiam a necessidade de observar também a saúde das pessoas que não foram infectadas pelo vírus, principalmente aquelas que pararam de trabalhar durante a pandemia. Destaca-se que pessoas fisicamente ativas podem estar mais suscetíveis a problemas de bem-estar durante o lockdown. Os formuladores de políticas que estipularam as restrições e medidas que visam conter o avanço da pandemia da COVID-19 podem se beneficiar da compreensão de tais implicações para a saúde e o bem-estar ${ }^{12}$, assim estimulando o teletrabalho e colaborando para que determinadas atividades laborais não sejam paralisadas.

Diante do exposto, questiona-se como os setores de gestão de pessoas aderiram ao teletrabalho como medida preventiva à contaminação da COVID-19. O objetivo do presente estudo foi analisar as evidências científicas disponíveis na literatura nacional e internacional sobre a gestão de pessoas atuantes no teletrabalho desenvolvido diante da medida preventiva (isolamento social) imposta durante a pandemia da doença COVID-19.

\section{MÉTODO}

Trata-se de um estudo de exploração qualitativa e descritiva dos dados da literatura, que permitiu a síntese dos estudos selecionados. É um método valioso de pesquisa pois, proporciona conhecimento por meio de um processo sistemático e rigoroso. Sua condução baseia-se em um rigor metodológico adequado e pertinente. As etapas deste método basearam-se de acordo com sugerido por autores ${ }^{13-14}$ :

$\checkmark$ Identificação do tema e ou elaboração da pergunta da revisão; existe evidências científicas sobre a gestão estratégica de pessoas atuantes no teletrabalho desenvolvido diante da medida preventiva imposta durante a pandemia da doença COVID-19;

$\checkmark$ Busca e seleção dos estudos primários de acordo com os critérios de inclusão e exclusão previamente estabelecidos; os critérios de inclusão consistiram em artigos originais completos e disponíveis na íntegra que retratassem a temática proposta no estudo em qualquer idioma e período;

$\checkmark$ Avaliação crítica dos estudos primários incluídos na revisão; estudos foram lidos e analisados na íntegra;

$\checkmark$ Interpretação e síntese dos resultados dos estudos encontrados; após a busca manual nas bases de dados, foram sintetizados de acordo com a estratégia de busca específica;

$\checkmark$ Síntese do conhecimento; divulgou-se nos resultados os conhecimentos adquiridos. 
O estudo foi desenvolvido entre os meses de abril e junho de 2020 , sendo que para a seleção das bases, realizou-se sorteio em um aplicativo online chamado sorteador, sendo selecionadas as bases PUBMED, Literatura Latino-Americana e do Caribe em Ciências da Saúde (LILACS) e Physiotherapy Evidence Database (PEDro) e na Biblioteca Eletrônica Científica Online (SCIELO). Utilizou-se as palavras-chaves em português: gestão de pessoas, teletrabalho, trabalho em home office, trabalho remoto, coronavírus, COVID-19 e em inglês:personnel management, telework, remote work, home office, Sarscov-2, COVID-19 e coronavírus. Para as palavras-chaves utilizadas, as combinações realizadas nesse estudo utilizaram o termo booleano "AND". Ainda utilizou-se o apoio de literatura especializada para complementar a discussão da temática.

Os critérios de inclusão estabelecidos foram ser artigo primário disponibilizado na íntegra, publicado nos idiomas inglês e português, em qualquer período e que abordava a temática proposta. As estratégias de busca seguiram o padrão específico de cada base consultada.

Para obtenção dos dados, utilizou-se uma planilha composta de itens relativos à identificação do artigo, tipo de estudo, nível de evidência das pesquisas, objetivos e principais resultados. Os níveis de evidência seguidos foram nível I (mais forte): revisão sistemática ou metanálise de ensaios clínicos randomizados controlados ou provenientes de diretrizes clínicas baseadas em revisões sistemáticas de ensaios clínicos randomizados controlados; nível II: pelo menos um ensaio clínico randomizado controlado bem delineado; nível III: ensaios clínicos bem delineados sem randomização; nível IV: estudos de coorte e de caso-controle bem delineados; nível V: revisão sistemática de estudos descritivos e qualitativos; nível VI: um único estudo descritivo ou qualitativo; nível VII: opinião de autoridades e/ou relatório de comitês de especialistas ${ }^{15}$.

Por ser uma revisão integrativa da literatura, este estudo dispensou submissão em Comitê de Ética em Pesquisa.

\section{RESULTADOS}

A coleta de dados foi realizada em duas etapas. A primeira consistiu na busca avançada nas bases de dados selecionadas, sendo encontrados 16 estudos, quando se empregou associações das palavras chaves. Após o processo de identificação dos artigos que obedeceram aos critérios de inclusão estabelecidos, realizou-se a exclusão de quatro estudos duplicados e após a prévia leitura de todos os títulos e resumos, selecionou-se quatro estudos para elegibilidade. Na segunda etapa, procedeu-se à leitura na íntegra desses estudos, sendo excluidos mais dois artigos que não contribuiram com a temática proposta, resultando na amostra de dois artigos.

A Tabela 1 apresenta a estratégia realizada para busca de estudos nas bases de dados selecionadas. 
Tabela 1. Estratégia de busca nas bases de dados. 2020.

\begin{tabular}{|c|c|c|}
\hline Local de busca & Estratégia de busca & $\begin{array}{l}\text { Número de artigos encontrados } \\
\text { na primeira busca }\end{array}$ \\
\hline LILACS & $\begin{array}{l}\text { Gestão de pessoas AND Teletrabalho } \\
\text { AND COVID-19 } \\
\text { Teletrabalho AND coronavirus } \\
\text { Gestão de pessoas AND trabalho em } \\
\text { home office } A N D \text { coronavírus, } \\
\text { Gestão de pessoas } A N D \text { coronavírus }\end{array}$ & 06 \\
\hline PUBMED & $\begin{array}{l}\text { Personnel Management AND Telework } \\
\text { AND Sars-cov-2 } \\
\text { Personnel Management AND Telework } \\
\text { AND COVID-19 } \\
\text { Personnel Management AND Telework } \\
\text { AND coronavirus } \\
\text { Personnel Management AND Remote } \\
\text { work AND Sars-cov-2 } \\
\text { Personnel Management AND Remote } \\
\text { work AND COVID-19-2 } \\
\text { Personnel Management AND Remote } \\
\text { work AND coronavirus }\end{array}$ & 10 \\
\hline PEDro & $\begin{array}{l}\text { Personnel Management AND Telework } \\
\text { AND Sars-cov-2 } \\
\text { Personnel Management AND Telework } \\
\text { AND COVID-19 } \\
\text { Personnel Management AND Telework } \\
\text { AND coronavirus } \\
\text { Personnel Management AND Remote } \\
\text { work AND Sars-cov-2 } \\
\text { Personnel Management AND Remote } \\
\text { work AND COVID-19-2 } \\
\text { Personnel Management AND Remote } \\
\text { work AND coronavirus }\end{array}$ & 00 \\
\hline SCIELO & $\begin{array}{l}\text { Gestão de pessoas AND Teletrabalho } \\
\text { AND COVID-19 } \\
\text { Teletrabalho AND coronavirus } \\
\text { Gestão de pessoas AND trabalho em } \\
\text { home office } A N D \text { coronavírus }\end{array}$ & 00 \\
\hline
\end{tabular}

Fonte: Elaborado pelos autores (2020). 
A Figura 1 mostra a representação gráfica do fluxo da seleção dos artigos, constando o número de estudos encontrados inicialmente nas bases de dados, as exclusões e a amostra final.

Figura 1. Distribuição total dos estudos, segundo as bases de dados: LILACS e PUBMED,2020.

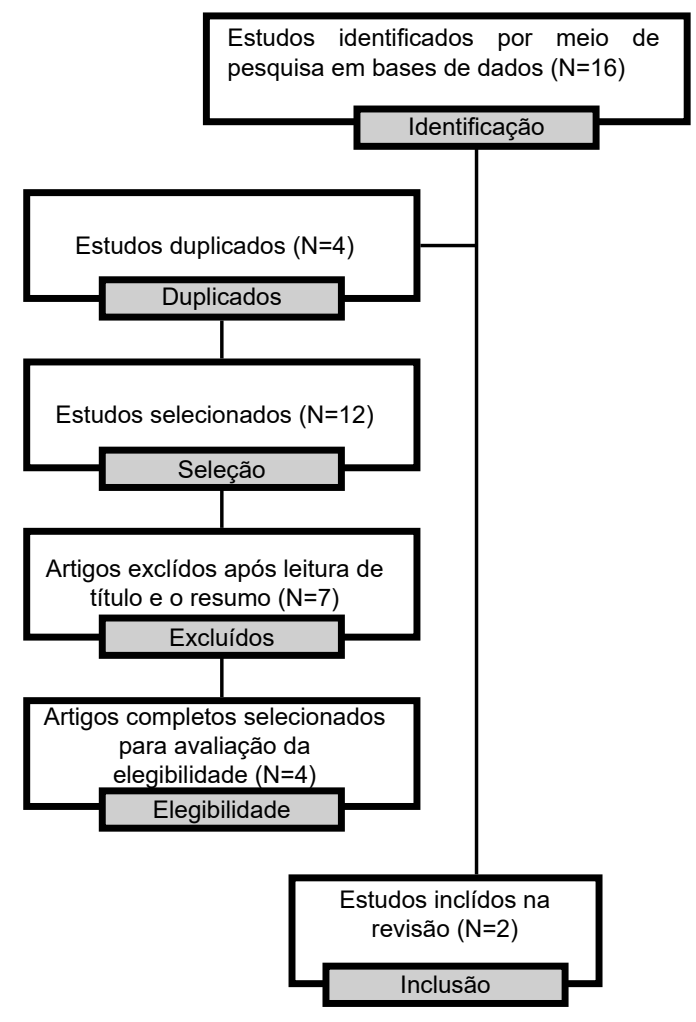

Fonte: Elaborado pelos autores (2020).

A Tabela 2 apresenta os dois estudos que fizeram parte dos achados para essa pesquisa.

Tabela 2. Estudos incluídos no estudo, segundo as bases de dados: LILACS e PUBMED,2020.

\begin{tabular}{ll}
\hline \multicolumn{1}{c}{ Estudo } & \multicolumn{1}{c}{ Título } \\
\hline Estudo 1 & A Web- and App-Based Connected Care Solution for COVID-19 In- \\
& and Outpatient Care: Qualitative Study and Application Development. \\
Autores & Schinköthe T, Gabri MR, Mitterer M, Gouveia P, Heinemann V, Har- \\
& beck N, Subklewe M. \\
Periódico & JMIR public health and surveillance. \\
Ano & 2020 \\
Nível de evidência & VI \\
Objetivo & Implementar uma ferramenta gratuita baseada na Web e em aplicati- \\
& vos para avaliação de pacientes, a fim de auxiliar os profissionais de \\
& saúde que trabalham no atendimento aos pacientes com COVID-19. \\
Método & Estudo realizado com entrevistas não estruturadas, os participantes \\
& das pesquisas foram médicos atuantes na Argentina, Alemanha, Irã, \\
& Itália, Portugal, Suíça e Estados Unidos,
\end{tabular}


Resultados

Conclusão

Os médicos perceberam a importância da telemedicina e soluções de atendimento, conectados para melhorar rapidamente $o$ atendimento aos pacientes; além do fato de haverem médicos infectados em quarentena atendendo por meio desta modalidade.

Esse processo de telemedicina foi ágil e interativo e complementa o trabalho dos médicos, mantendo em segurança todos os envolvidos.

$\begin{array}{ll}\text { Estudo } 2 & \text { How Are We Facing It? Dispatches From Pathology Residents in a } \\ & \text { COVID-19 Lombardy Hospital. } \\ \text { Autores } & \text { Cieri M, De Carlo C, Valeri M, Belsito V, Lancellotti C, Roncalli M, Co- } \\ & \text { lombo P } \\ \text { Periódico } & \text { Front. Public Health } \\ \text { Ano, } & 2020 . \\ \text { Nível de evidência } & \text { VII. } \\ \text { Objetivo } & \begin{array}{l}\text { Ilustrar as principais mudanças e medidas de segurança adotadas em } \\ \text { um departamento de patologia de um hospital da Lombardia na Itália }\end{array} \\ \text { Método } & \begin{array}{l}\text { Relato de experiência, no qual obteve relato de médicos envolvidos no } \\ \text { setor de patologia de um hospital da Lombardia na Itália no período } \\ \text { pandêmico desse país. }\end{array} \\ \text { Resultados } & \begin{array}{l}\text { Devido ao número decrescente de procedimentos cirúrgicos, o de- } \\ \text { partamento de Patologia adotou uma política de redução de trabalho } \\ \text { presencial, permitindo que os patologistas trabalhassem remotamente } \\ \text { durante a pandemia. }\end{array} \\ \text { A adoção do trabalho remoto permitiu garantir um serviço no depar- } \\ \text { tamento respeitando as regras de distanciamento social, encarando } \\ \text { uma nova organização de trabalho e lidando com a tecnologia. De } \\ \text { fato, a tecnologia usada para o trabalho inteligente, a fim de aumentar } \\ \text { o distanciamento social e o uso da tecnologia digital, encontra novo e } \\ \text { interessante papel na rotina desses médicos, na realização de diag- } \\ \text { nósticos com experiência em treinamento remoto. }\end{array}$

Fonte: Elaborado pelos autores (2020).

\section{DISCUSSÃO}

Devido às estratégias de prevenção e o controle da propagação da COVID19, no contexto do mundo do trabalho, muitas pessoas passaram a realizar suas atividades laborais nas suas residências ${ }^{16}$. Esta modalidade de trabalho tem sido cada vez mais frequente e caracteriza-se pela realização do trabalho de maneira remota, por meio de 
tecnologias de informação e comunicação, sendo conceituado com teletrabalho ${ }^{17}$. $O$ teletrabalho hoje é uma realidade e é até uma necessidade. Diante desse cenário de crise, o teletrabalho pode significar uma estratégia de controle da pandemia, uma vez que pode diminuir as chances de proliferação do vírus.

O primeiro estudo selecionado nesta revisão foi desenvolvido com profissionais médicos atuantes na Argentina, Alemanha, Estados Unidos, Irã, Itália, Portugal e Suíça e utilizou entrevistas não estruturadas. A equipe de gestão de pessoas, para diminuir os riscos de infecção da COVID-19, traçou metas para as soluções de atendimento conectado, cuja estratégia envolvia o monitoramento remoto de pacientes, por meio do teletrabalho. Assim, foi possível manter comunicações seguras entre médicos e os pacientes, mantendo medidas de prevenção frente a essa emergência de Saúde Pública ${ }^{18}$.

Os profissionais de medicina por meio do trabalho remoto compreenderam que poderiam melhorar rapidamente o atendimento ao seu paciente, bem como diminuir as chances de contágio da doença COVID-19. Portanto, foram implementados no teletrabalho, rastreamento remoto de pacientes acometidos com doenças crônicas e ainda, os médicos infectados pela doença que se mantinham em isolamento social puderam atuar por meio do teletrabalho ${ }^{18}$.

Outros benefícios importantes encontrados nesta pesquisa desenvolvida nestes sete países, foram a redução de carga de trabalho dos profissionais de saúde e as interações sem contato físico entre médicos e pacientes, com uso de um aplicativo de smartphone. Desse modo, nos países que optaram em utilizar o aplicativo, este se tornou uma ferramenta ágil e interativa que complementava o trabalho dos médicos, melhorando o atendimento e a segurança das pessoas infectadas pela COVID-19 ${ }^{18}$. Essa informação é muito importante e pode colaborar para a diminuição da exposição do contágio da doença, principalmente entre os profissionais de saúde.

Para o destaque referente as gestões laborais, ressalta-se que é possível no teletrabalho a conexão com seu trabalho por meio de tecnologias,softwares, ligações de banda larga residenciais entre outras, que permitem menores custos para asinstituições ${ }^{19}$ e nesse período pandêmico é uma ótima ferramenta. Chama-se atenção para que o teletrabalho quebra barreiras entre países, somando-se a um grande acesso de informação, educação, treinamento e emprego ${ }^{19}$.

Outro estudo mencionou o pico da doença COVID-19 no final de fevereiro, registrado no Serviço Nacional de Saúde da Itália na região da Lombardia, onde esta infecção viral altamente patogênica, apresentando inúmeros desafios para os profissionais de saúde. Os departamentos de patologia foram envolvidos na reorganização do gerenciamento de pessoas, devido ao número decrescente de procedimentos cirúrgicos e a política para reduzir o pessoal nos locais de trabalho foi implementada. Permitiu-se então que os patologistas 
trabalhassem de forma remota durante a pandemia. A equipe de patologia recebeu um treinamento específico para aderência às novas normas, para que remodelassem sua rotina diária, mantivessem distâncias seguras, com isso a vida diária desses profissionais mudou drasticamente ${ }^{20}$.

Os residentes patologistas italianos do Departamento de Patologia Cirúrgica, compartilharam sua experiência nesse novo cenário e ilustraram as principais mudanças e medidas de segurança adotadas no hospital da Lombárdia. Uma das estratégias realizadas foi orientar os consultores sêniores e estudantes de medicina a não comparecerem no laboratório. Os patologistas sêniores receberam orientações tecnológicas para trabalharem remotamente e agendavam uma atividade de trabalho inteligente duas vezes por semana, contribuindo para medidas gerais de segurança. Como consequência, foi implementada a patologia digital, que anteriormente era usada apenas para fins de pesquisa e a partir da pandemia foi introduzida para treinamento de residentes em histopatologia, aulas virtuais de citologia, quadros de tumores multidisciplinares e reuniões interinstitucionais ${ }^{20}$.

Adotar uma nova tecnologia de trabalho foi um desafio inicialmente, mas devido a atual situação, houve a adesão e empenho dos médicos patologistas na utilização das ferramentas digitais com entusiasmo. Os médicos revisaram imagens pelomonitor, fazendo anotações e obtendo fotos quando necessário. Além disso, o compartilhamento de links, tabelas e gráficos nos diálogos entre as equipes foi simples e houve fácil adaptação ${ }^{20}$. Assim como no caso de médicos, é interessante mencionar que outros profissionais ao aderir o teletrabalho podem contar com o processo de ensino-aprendizagem ${ }^{21}$.

Um estudo realizado nos Estados Unidos, que não apareceu nas buscas de dados sorteadas nesse estudo, ressaltou que o College of American Pathologist e os Centros de Serviços Medicare e Medicaid, já haviam solicitado ao Congresso que apoiasse essa solicitação para que os patologistas desse país atuassem remotamente durante a pandemia da COVID-19. Além disso, a gestão de pessoas College of American Pathologist, declarou que todos os laboratórios deveriam ter o poder de determinar o que é melhor para seus trabalhadores no gerenciamento dessa pandemia ${ }^{22}$. Desse modo, tanto o College of American Pathologist quanto o Departamento de Patologia Cirúrgica da Lombardia, buscaram a adoção dessa política para a segurança do pessoal ${ }^{22}$.

Enfim, os residentes de patologia concluíram que, apesar dos vários problemas na instituição causados pela crise da pandemia, eles tiveram a oportunidade de manter o trabalho, respeitando as regras de distanciamento social, enfrentando uma nova organização de trabalho e lidando com a tecnologia necessária para o momento. De fato, a tecnologia usada para o trabalho remoto a fim de aumentar o distanciamento social e o uso da patologia digital, encontra um novo e interessante papel na rotina, na 
realização de diagnósticos com experiência de intercâmbio entre residentes e tutor e em treinamento remoto ${ }^{20}$.

Destaca-se que é muito importante a equipe de gestão de pessoas conhecer as características do telebrabalho que podem ser positivas ou negativas, isto é, devem se atentar a isso na relação contratual. No teletrabalho as novas tecnologias possibilitam ao trabalhador, muitas vezes, a convivência com a família e a terem outras relações pessoais. Por outro lado, a condição poderá levá-lo a um convívio profissional prejudicado, o qual é elemento contributivo na construção da felicidade e contentamentos ${ }^{19}$.

Nota-se que o isolamento do teletrabalhador pode levar a quadros de adoecimentos de natureza mental ${ }^{20}$, levando em consideração que algumas pessoas têm maiores propensões a desenvolverem o estresse, a ansiedade, a depressão e a síndrome deburnout, podendo gerar assim, afastamentos e acidentes de trabalhos. Diante do exposto, é de extrema importância a responsabilidade e dever do empregador em observar a compatibilidade do teletrabalhador com esse tipo de modalidade de trabalho ${ }^{19}$.

Vale a pena mencionar, que o incremento nas relações virtuais entre o empregador e o empregado são alvo de discussões ${ }^{16}$. O teletrabalho apresenta desvantagens, devido ao isolamento (que nessa pandemia é fundamental), a perda do contato direto com seus colegas de trabalho e superiores, a pouca criatividade nas atividades executadas; além de longas jornadas de trabalhos ${ }^{21}$.

Ressalta-se a necessidade de intensificar pesquisas que apresentem resultados de gestão de pessoas no teletrabalho durante a pandemia da COVID-19 e que sejam capazes de produzir maiores evidências científicas, já que identificou-se poucas pesquisas sobre a temática, deve-se elencar medidas de segurança e saúde ocupacional, visto que ainda não se sabe quando será o final da pandemia. É preciso que a gestão de pessoas no teletrabalho executado de forma emergencial durante a pandemia, observe e proporcioneo mínimo de conforto e segurança no trabalho necessário, para evitar o surgimento de doenças ocupacionais.

O estudo evidenciou que há necessidade de dar atenção e formular políticas e potenciais cuidados e investigações nesse âmbito. Complementa trazendo que a temática merece ser alvopara estudos futuros. Espera-se que os resultados desse estudo auxiliem no planejamento adequado do teletrabalho visando a saúde dos trabalhadores.

Assim, nos artigos selecionados neste estudo surgiram pontos importantes para o desenvolvimento de atividades médicas contemplando as necessidades do trabalho e a abordagem referente aos aspectos emocionais e saúde mental dos profissionais médicos no teletrabalho. 
Embora não tenha sido intencional conhecer o teletrabalho em profissionais de saúde, foram estes os profissionais pesquisados nos resultados obtidos por meio das buscas em bases de dados. Chama-se atenção que os estudos encontrados possuem nível de evidências considerados fracos, sugere-se a criação de evidências mais robustas relacionadas à temática, fazendo necessário despertar o olhar da equipe de gestão de pessoas. Como limitação, desse estudo aponta-se o número pequeno de artigos encontrados nas bases de dados selecionadas.

\section{CONCLUSÃO}

Apenas dois artigos foram relacionados com a temática proposta. Ambos estudos eram internacionais e mostraram a gestão estratégica de pessoas para o teletrabalho em profissionais de saúde. A gestão estratégica de pessoas foi implementada no teletrabalho de profissionais de saúde, adaptando com ferramentas digitais e tecnológicas para a atividade laboral, bem como atingiram resultados positivos, no qual médicos em isolamento social puderam continuar atuando para atender a demanda necessária, também puderam melhorar $\mathrm{o}$ atendimento e a segurança dos evolvidos.

\section{REFERÊNCIAS}

1. Wu Y, Xu X, Chen Z, Duan J, Hashimoto K, Yang L et al. Nervous system involvement after infection with COVID-19 and other coronaviruses. Brain, behavior, and immunity [Internet]. 2020 18(22) [acesso em 2020 jul 02]; Disponível em: https://doi.org/10.1016/j.bbi.2020.03.031

2. Wang J, Qi H, Bao L, Li F, Shi Y. A contingency plan for the management of the 2019 novel coronavirus outbreak in neonatal intensive care units. The Lancet Child and Adolescent Health [Internet]. 2020 4(4) [acesso em 2020 jul 02]; Disponível em: http://dx.doi.org/10.1016/S2352-4642(20)30040-7.

3. Xu Y. Unveiling the Origin and Transmission of 2019-nCoV. Trends in Microbiology [Internet]. 2020a 28(4). [acesso em 2020 jul 02]; Disponível em: https://doi.org/10.1016/j.tim.2020.02.001.

4. Di Pasquale G. Coronavirus COVID-19: Quali implicazioni per la Cardiologia? Giornale Italiano di Cardiologia [Internet]. 202021(4) [acesso em 2020 jun 25];. Disponível em:https://www.giornaledicardiologia. it/articoli.php?archivio=yes\&vol_id=3328\&id=32981.

4. Singhal T. A Review of Coronavirus Disease-2019 (COVID-19). Indian Journal of Pediatrics [Internet]. 2020 87(4) [acesso em 2020 jul 01];.Disponível em: <https://www.ncbi.nlm.nih.gov/pmc/articles/PMC7090728/>.

6. Xu J, ChenY, ChenH, CaoB. 2019 novel Coronavirus outbreak: a quiz or final exam? Frontiers of Medicine [Internet]. 2020b [acesso em 2020 jul 02]; Disponível em:https://www.researchgate.net/ publication/340071323_2019_novel_Coronavirus_outbreak_a_quiz_or_final_exam.

7. Wang D, Hu B, Hu C, Zhu F, Liu X, Zhang J et al. Clinical characteristics of 138 hospitalized patients with 2019 novel coronavirus-infected pneumonia in Wuhan, China. JAMA [Internet]. 2020b [acesso em 2020 jul 01]; Disponível em: https://pubmed.ncbi.nlm.nih.gov/32031570/.

8. Zhang J, Dong X, Cao YY, Yuan YD, Yang Y, Yan Y et al. Clinical characteristics of 140 patients infected with SARS-CoV-2 in Wuhan, China. Allergy [Internet]. 2020a 2(19). [acesso em 2020 jul 01]; Disponível em:https://onlinelibrary.wiley.com/doi/full/10.1111/all.14238.

9. Zhou F, Yu T, Du R, Fan G, Liu Y, Liu Z et al. Clinical course and risk factors for mortality of adult inpatients with COVID-19 in Wuhan, China: a retrospective cohort study. Lancet [Internet]. 2020;395(10229): [acesso em 2020 jul 10]; Disponível em:https://www.thelancet.com/journals/lancet/article/PIIS0140-6736(20)30566-3/fulltext . 
10. De Oliveira ECB, De Lima MAV, Bryto KKC. A relevância da gestão estratégica de pessoas no ambiente escolar. Revista de Administração e Contabilidade-RAC [Internet]. 2019; 6(12).

11. Ahmed F, KimS, Nowalk MP, King JP, VanWormer JJ, Gaglani M et al.,Paid leave and access to telework as work attendance determinants during acute respiratory illness, United States, 2017-2018. Emerging Infectious Diseases [Internet]. 2020. [acesso em 2020 jun 11]; Disponível em: https://www.scielo.br/scielo. php?script=sci_arttext\&pid=S1981-77462020000200501\&lng=pt\&nrm=iso\&tlng=pt .

12. Zhang SX. Wang Y, Rauch A, Wei FUnprecedented disruption of lives and work: Health, distress and life satisfaction of working adults in China one month into the COVID-19 outbreak. Psychiatry Res [Internet]. 2020. [acesso em 2020 jul 01]; Disponível em:https://www.sciencedirect.com/science/article/pii/ S0165178120306521.

13. Soares CB, Hoga LAK, Peduzzi M, Sangaleti C, Yonekura T, Silva DRAD. Revisão integrativa: conceitos e métodos utilizados na enfermagem. Revista da Escola de Enfermagem da USP. 2014; 48(2).

14. Mendes KDSS, Silveira RCCP, Galvão CM. Revisão integrativa: método de pesquisa para a incorporação de evidências na saúde e na enfermagem. Texto \& contexto enfermagem. 2008; 17(4).

15. Stetler CB, Rucki D, Broughton S, Corrigan S,Fitzgerald B, Giuliano,K,.et al. Utilization-focused integrative reviews in a nursing service. Appl Nurs Res. 1998; 11(4):.

16. Geovrick JGJ. O teletrabalho em angola. [s. I.], 2020.

17. Rocha CTM, Amador FS. O teletrabalho: conceituação e questões para análise. Cadernos EBAPE. BR, 2018; 16(1).

18. SchinkötheT, Gabri MR, Mitterer M, Gouveia P, Heinemann V, Harbeck N, et al,. A Web- and AppBased Connected Care Solution for COVID-19 In- and Outpatient Care: Qualitative Study and Application Development. JMIR Public Health Surveill [Internet]. 2020. [acesso em 2020 jun 25]; 1;6(2). Disponível em: https://publichealth.jmir.org/2020/2/e19033/.

19. Lucena JP. O teletrabalho e o controle do trabalhador à distância. O teletrabalho e o controle do trabalhador à distância, 2015

20. Cieri M, De Carlo C, Valeri M, Belsito V, Lancellotti C, Roncalli M, et al. How Are We Facing It? Dispatches From Pathology Residents in a COVID-19 Lombardy Hospital. Frontiers in Public Health [Internet]. 2020. Disponível em: http://dx.doi.org/10.3389/fpubh.2020.00259

21. Ferreira JP, Oliveira RMS. O teletrabalho no contexto da educação a distância. Multifaces: Revista de Ciência, Tecnologia e Educação. 2018; 1(1).

22. College of american pathologists. Secures Remote Work Waiver for Pathologists [Internet]. 2020 [acesso em 2020 jul 08]; Disponível em: https://www.cap.org/advocacy/latest-news-and-practice-data/ march-26-2020.

RECEBIDO: $16 / 07 / 2020$

ACEITO: 07/10/2020 